\title{
Posteriorly positioned femoral grafts decrease long-term failure in anterior cruciate ligament reconstruction, femoral and tibial graft positions did not affect long-term reported outcome
}

\author{
Tim T. C. R. de Mees ${ }^{1}\left[\right.$ ] Max Reijman ${ }^{1}$ · Jan Hendrik Waarsing ${ }^{1} \cdot$ Duncan E. Meuffels $^{1}$
}

Received: 1 July 2021 / Accepted: 5 January 2022 / Published online: 2 February 2022

(C) The Author(s) 2022

\begin{abstract}
Purpose To investigate the effect that femoral and tibial tunnel positions have on long-term reported and clinical outcome and to identify a safe zone based on favourable outcome.

Methods Seventy-eight patients from a previous randomised controlled trial were included and were followed with a mean follow-up of 11.4 years. All patients had primary trans-tibial anterior cruciate ligament reconstruction performed. The femoral and tibial tunnel positions were visualised and translated in percentages with three-dimensional computed tomography post-operatively. There were 3 separate outcome variables: patient-reported outcome measured with the IKDC Subjective Knee Form, overall failure, and radiographic osteoarthritis. The correlation between tunnel aperture positions and outcome was determined with multivariate regression. The area with best outcome was defined as the safe zone and was determined with Youden's index in conjunction with receiver operating characteristics.

Results No significant relationship was found between tunnel aperture positions and IKDC Subjective Knee Form at 10-year follow-up. The posterior-to-anterior femoral tunnel aperture position parallel to Blumensaat line showed a significant relationship ( $p=0.03$ ) to overall failure at 10-year follow-up. The mean posterior-to-anterior tunnel position of the group that did not fail was $37.7 \%$ compared to $44.1 \%$ in the overall failure group. Femoral tunnel apertures placed further anteriorly had more overall failures at long-term. The cut-off point lies at $35.0 \%$ from posterior-to-anterior parallel to Blumensaat. Of the 16 overall failures, $15(93.8 \%)$ were placed further anteriorly than the cut-off point. No significant relationship was found between tunnel aperture positions and radiographic osteoarthritis.

Conclusion Femoral and tibial tunnel positions were not associated with long-term patient-reported outcome and radiographic osteoarthritis. Long-term overall failure was more frequently seen in patients with a more anteriorly placed femoral tunnel. This study identified a safe zone located at the most posterior $35 \%$ of the femoral condyle parallel to Blumensaat. This knowledge offers guidance to surgeons to operate more precisely and accurately and reconstruct a long-lasting graft. Level of evidence Level III.
\end{abstract}

Keyword Anterior cruciate ligament reconstruction · Trunnel positioning · Graft placement · Long-term outcome · Graft failure $\cdot$ IKDC

Tim T. C. R. de Mees

t.demees@erasmusmc.nl

1 Department of Orthopaedic Surgery, Erasmus MC, University Medical Center Rotterdam, Dr. Molewaterplein 40, 3015 GD Rotterdam, The Netherlands

\section{Introduction}

Achieving anatomical resemblance in graft positioning is an important goal in anterior cruciate ligament (ACL) reconstruction, but is also a goal of great complexity. The surgeon has to consider numerous factors whilst operating within the anatomic boundaries of the femoral intercondylar notch and tibial eminences. Of these factors, tunnel positioning is most essential to achieve an anatomic graft position and an excellent ACL reconstruction [2, 3, 9, 20, 21, 23, 24, 28, 36, 40, 41, 43, 46, 51, 57]. 
Improper graft position leads to decreased patientreported outcome, increased knee laxity and increased failure rate $[3,20,21,28,36,43,46]$. The anatomical explanations for this are graft impingement in the roof of the intercondylar notch, excessive graft forces, graft laxity, and tunnel widening [2, 17, 24, 28, 32, 36, 55]. Several studies found an association between tunnel positions and outcome, especially at short term $[2,3,9,21,24,36,43]$. Principally, the tunnel positions should be anatomically oriented to create optimal stability. The femoral tunnel aperture position resembles the anatomy best if it is placed posteriorly and around middle-height in the lateral condyle [5, 31, 36, 39, $55,60]$. The tibial tunnel aperture position resembles the ACL best when placed between the eminences and slightly more anteriorly than the midline $[4,8,15,28,31,32,39$, 47].

However, between all these associations, there is no consensus yet about which exact tunnel positions resemble the anatomy best. The most precise visualisation of these tunnel aperture positions can be obtained with three-dimensional computed tomography (3D-CT). This imaging technique provides a $100 \%$ visualisation with excellent intra- and interobserver reliability $[25,30]$.

The long-term functioning of a graft, which is measured with different long-term outcome parameters, is essential to retain patient satisfaction and high activity levels [13, 44]. Long-term outcome has different dimensions and exists of patient-reported outcome, clinical outcome (such as knee laxity) and graft failure and revision (due to graft ruptures). There is a limited number of studies that linked this long-term outcome to radiographically assessed tunnel positions [15, 41, 50]. However, the relation between longterm outcome and precisely visualised femoral and tibial tunnel aperture positions with 3D-CT has not been studied yet. The purpose of our study was to investigate the effect that femoral and tibial tunnel positions have on long-term reported and clinical outcome and to identify a safe zone based on favourable outcome. The hypothesis is that femoral and tibial tunnel apertures placed closer to the anatomic ACL positions show more favourable long-term outcome compared to non-anatomically placed tunnel positions [5, 7, $11,47]$. Knowledge about the relation between exact tunnel positions and long-term outcome is clinically relevant for surgeons to operate more precise and accurate and reconstruct a long-lasting graft.

\section{Materials and methods}

This is a retrospective cohort study with a prospective follow-up examination at 10 years post-operatively. All patients received a primary ACL reconstruction with direct post-operative visualisation by $3 \mathrm{D}-\mathrm{CT}$ scan in the University
Medical Centre between January 2007 and December 2009. The participants in this study were enrolled in a previous double-blind, randomised controlled trial (RCT). The RCT compared the precision and accuracy of computer-assisted surgery (CAS) with conventional ACL reconstruction [31]. The Medical Ethics Committee of the Erasmus University Medical Centre approved the study protocol of the RCT and the follow-up study (METC-2006-223 and METC2019-0369) and all participants provided written informed consent.

\section{Inclusion of patients}

Patients were included if they were 18 years or older, were eligible for primary ACL reconstruction and received a postoperative 3D-CT. The occurrence or absence of meniscal injury was registered and treated when necessary. Patients were excluded if they had additional posterior cruciate ligament or collateral ligament injury or if they had insufficient comprehension of Dutch or English. Patients were lost to follow-up if they were unreachable, unable or unwilling to comply with the 10-year post-operative follow-up.

\section{Surgery}

Two fellowship-trained orthopaedic surgeons, who both perform more than 100 ACL reconstructions annually, performed all ACL reconstructions of this study. The ACL reconstruction was performed using an arthroscopic, single-incision, single-bundle, trans-tibial surgical technique, using either bone-patella-tendon-bone (BPTB) or a looped semi-tendinosus, gracillis autograft. The choice for either graft was made individualised per patient by the surgeon pre-operatively depending on specific athletic demands and other knee comorbidities. The graft diameters ranged from 8 to $11 \mathrm{~mm}$. Patients received conventional surgery or CAS, based on randomisation in the previous RCT. CAS was performed using a stand-alone infrared controlled computer (Version 1.0, Brainlab, München, Germany). This system uses per-operatively acquired radiographs of the knee. These are then used to template the femoral and tibial tunnel position in the computer.

The conventional femoral and tibial bone tunnels were positioned within the native anatomic ACL footprint. The aimed position of the tibial tunnel aperture in patients operated with CAS was located at $44 \%$ of the anterior-to-posterior length of the tibial plateau between the eminences $[39,47]$. The aimed femoral tunnel position with CAS was located at the origin of the anteromedial bundle. In the posterior-to-anterior direction, the aimed femoral aperture position was located at $24.8 \%$ measured parallel to the Blumensaat line. In the superior-to-inferior direction, the aimed 
femoral aperture position was located at $28.5 \%$ of the height perpendicular to the Blumensaat line [5].

\section{Outcome measures}

Patients were invited to fill in questionnaires and to visit the outpatient clinics for physical examination pre-operatively, at 2-year and 10-year follow-up, besides their regular postoperative follow-up. The questionnaires could be filled in either online or on paper. Physical examination consisted of manual and arthrometric laxity measurement. Arthrometric laxity measurement was performed using the KT-1000 (Medtronic, Cal. U.S.A.). The same experienced orthopaedic surgeon examined all patients pre-operatively, at 2-year, and 10-year follow-up, blinded for tunnel positions. Ipsilateral graft ruptures, contralateral ACL ruptures and other adverse events were registered for both knees.

There were two primary outcomes in this study: the absolute score of the International Knee Documentation Committee Subjective Knee Form (IKDC-SKF) and the overall failure at 10-year follow-up. The IKDC-SKF, provides a responsive outcome measurement of patients' perception of symptoms, knee function and ability to participate in sports activities after ACL reconstruction $[18,56]$. The IKDCSKF is a valid tool for patients with ligament or meniscal ruptures with good test-retest reliability, with an interclass correlation between 0.87 and 0.95 [14]. Overall failure was defined if one or more of the following criteria were present: a visualised graft rupture on MRI or revision surgery during follow-up (described as 'graft failure'), knee laxity during physical examination (Lachman $\geq 2+$, pivot shift $\geq 2+$, KT-1000 difference $\geq 4 \mathrm{~mm}$ or an IKDC objective score of C or D) or an IKDC-SKF score $<50$. The secondary outcome was radiographic osteoarthritis. Weight-bearing anteroposterior and lateral radiographs of both knees were made at 10-year follow-up. These were graded with the Kellgren and Lawrence (K\&L) classification for osteoarthritis by two researchers, blinded for patient allocation [22]. Radiographic osteoarthritis was defined as a $K \& L$ score of $\geq$ grade 2 on the operated knee.

\section{Measurement of the tunnel aperture positions}

The CT scan of the operated knee was made within $72 \mathrm{~h}$ post-operatively in all patients. A 64 channel multi-slice technology CT-scanner (Somatom, Siemens Medical Solutions, Forchheim, Germany) with helical acquisition in $1.0 \mathrm{~mm}$ sections $(120 \mathrm{kV}, 160 \mathrm{mAs}$, rotation time $1.0 \mathrm{~s})$ was used to determine the tunnel positions. The knee CT imaging was performed from the top of the suprapatellar pouch to the superior tibial and fibula diaphysis, post-operatively. The data were then transferred and blinded for patient, into the 3-dimensional measurement software. (MeVisLab Version 2.0, MeVis Medical solutions AG, Bremen, Germany).

Measurement of the three-dimensional images was performed by a radiologist blinded for patient allocation. The anatomic coordinate axis method was used for measurement $[25,30,52]$. Using the three-dimensional tri-axial properties of the desktop version of $\mathrm{MeVis}$, the contour of the femur (the intra-condylar axis and medial side of the lateral femoral condyle) and tibia (circumference of the tibial plateau) was mapped. The apertures of the femoral and tibial tunnel were mapped using the centre of the tunnel opening. All these measurements were performed and translated into a true sagittal view of the femoral condyle and a true transversal view of the tibial plateau. The femoral and tibial tunnel aperture positions were then translated into percentages of respectively the femoral condyle and the tibial plateau (Fig. 1).

\section{Statistical analysis}

Statistical analysis was performed with the use of SPSS software (IBM SPSS Statistics for Windows, Version 25.0. Armonk, NY: IBM Corp.). The Shapiro-Wilk analysis was performed to test for normal distribution of variables. Paired $t$ tests were executed to test the changes in patientreported outcome between baseline and 10-year follow-up. The intra-class correlation coefficients (ICC) were calculated to determine inter-observer reliability for tunnel position measurement. Linear regression analyses were performed to determine the relation between IKDC-SKF score (dependent variable) and the different tunnel aperture positions (independent variables). Logistic regression analyses were performed to determine the relation between the dichotomous dependent variables (overall failure and osteoarthritis) and tunnel aperture positions (independent variables). There was adjusted for the following variables: sex, Body Mass Index (BMI), type of surgery (computer-assisted or conventional), type of graft (bone-patella-tendon-bone or hamstring), meniscal tears, chondral defects (both observed per-operatively) and pre-operative Lachman score. There was also specifically adjusted for pre-operative IKDC-SKF and pre-operative grade of osteoarthritis in the analysis of respectively IKDC-SKF and osteoarthritis. In order to identify the safe zone for tunnel aperture positions, we performed the Youden's index in conjunction with receiver operating characteristic (ROC) analysis. A $p$ value less than 0.05 was considered statistically significant. $P$ values, $95 \%$ confidence intervals $(95 \% \mathrm{CI})$ and odds-ratios were reported with two decimals, all other values were reported with one decimal in tables and in text. No sample size calculation was performed since there was a fixed study population because it was the follow-up of a previous RCT. 


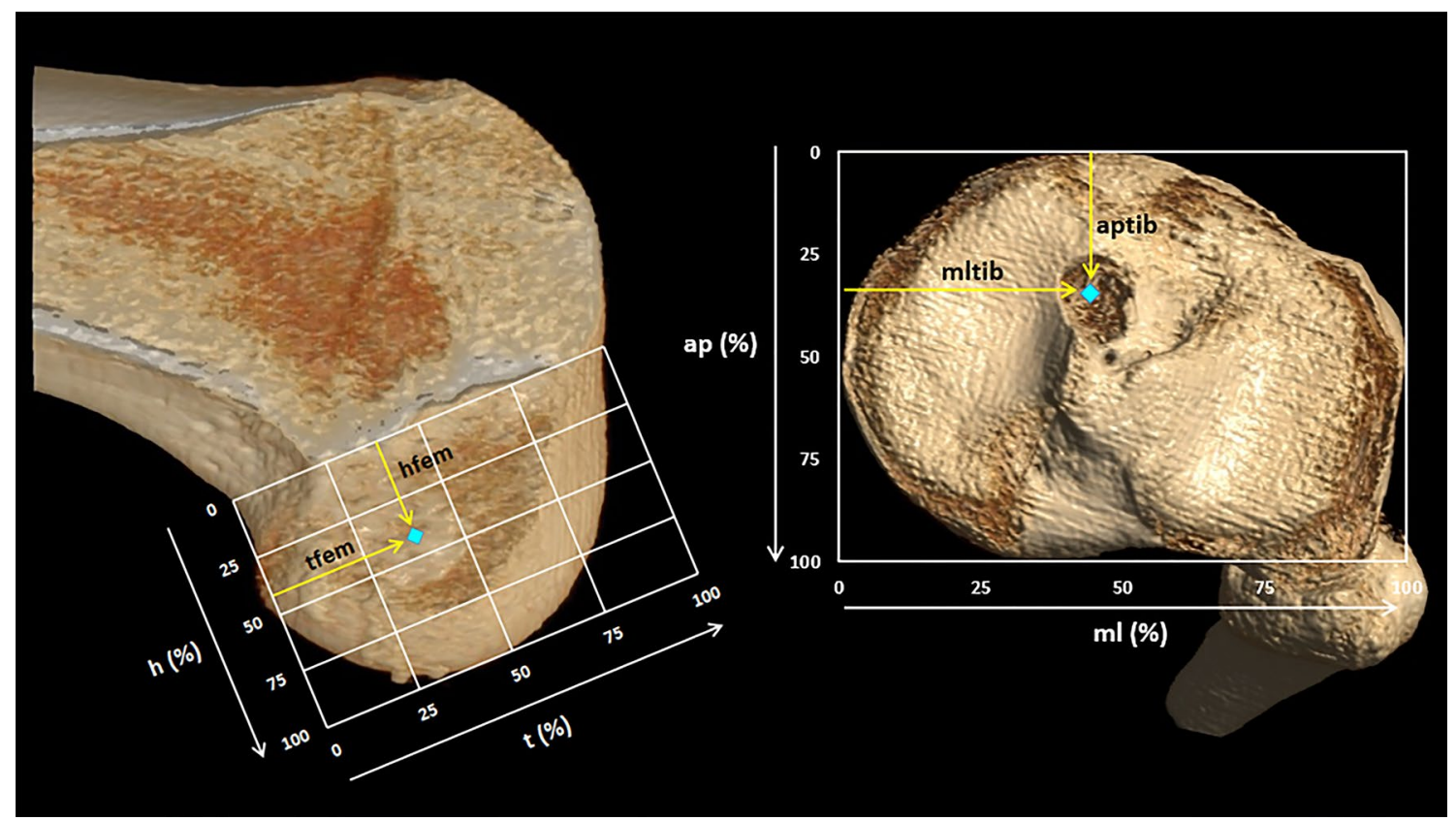

Fig. 1: 3D-CT view of the medial side of the lateral femoral condyle with the quadrants of Bernard (left) and the tibial plateau (right). Blue dot: Example tunnel aperture position. Femur: h: the total superior-inferior height of the quadrants, starting at $0 \%$ perpendicular at the Blumensaat line and ending at $100 \%$ at the end of the femoral condyle. $\mathrm{t}$ : the total posterior-to-anterior length of the quadrants, starting at $0 \%$ at the most posterior part of the condyle, parallel to Blumensaat and ending at $100 \%$ at the most anterior part. tfem: posterior-to-inferior position of the tunnel aperture, expressed in a percentage of t. hfem: superior-inferior position of the tunnel aperture,

\section{Results}

\section{Study population and baseline characteristics}

Of the 98 patients that received primary ACL reconstruction with direct 3D-CT visualisation, 78 patients reported the IKDC-SKF at 10-year follow-up and were included in the primary analysis. A flowchart of the patients in the study can be seen in Fig. 2.

The mean follow-up duration was 11.4 years and ranged from 9.7 years until 12.7 years. The baseline and per-operative characteristics are presented in Table 1. There were no significant differences in baseline and per-operative characteristics between the included and the excluded cases.

The femoral tunnel aperture positions ranged between 16.7 and $65.3 \%$ of the posterior-to-anterior distance and between 17.5 and $60.0 \%$ of the superior-inferior height. The tibial tunnel apertures ranged between 22.9 and $56.9 \%$ of the anterior-to-posterior distance and between 26.1 and $62.1 \%$ of the medial-to-lateral distance (Figs. 3A, 4A).

Inter-observer measurement of the three-dimensional tunnel aperture positions showed an ICC of the femoral expressed in a percentage of $\mathrm{h}$. Tibia: ap: the total anterior-to-posterior length of the quadrants, starting at $0 \%$ at the most anterior part of the tibial plateau and ending at $100 \%$ at the most posterior part of the tibial plateau. ml: the total medial-to-lateral length of the quadrants, starting at $0 \%$ at the most medial part of the tibial plateau and ending at $100 \%$ at the most lateral part of the tibial plateau. aptib: anterior-toposterior position of the tunnel aperture, expressed in a percentage of ap. mltib: medial-to-lateral position of the tunnel aperture, expressed in a percentage of $\mathrm{ml}$

positions of 0.90 (confidence interval (CI) $0.85-0.93$ ) and of the tibial positions was 0.99 (CI 0.98-0.99).

Median value and (interquartile range) is presented for age and BMI. Mean value (standard deviation) is presented for the tunnel aperture positions. Frequency (percentage) is presented for the other characteristics. $t$ fem $=$ posteriorto-anterior femoral tunnel position, $\mathrm{hfem}=$ superior-inferior femoral tunnel position, aptib $=$ anterior-to-posterior tibial tunnel position, $\mathrm{mltib}=$ medial-to-lateral tibial tunnel position.

\section{Primary outcome}

No significant associations were found between the tunnel aperture positions and the IKDC-SKF score at 10-year follow-up (Figs. 3A, 4A and Table 2). Additionally, also no significant associations between the tunnel aperture positions and the IKDC-SKF score at 2-year follow-up were found.

The IKDC-SKF showed significant improvement between the pre-operative measurement and 10-year follow-up. The mean IKDC-SKF score increased from 63.7 ( \pm 14.2$)$ preoperatively to $77.5( \pm 16.1)$ at 10 -year follow-up. This increase of 13.8 points was statistically significant $(p=0.00)$ 


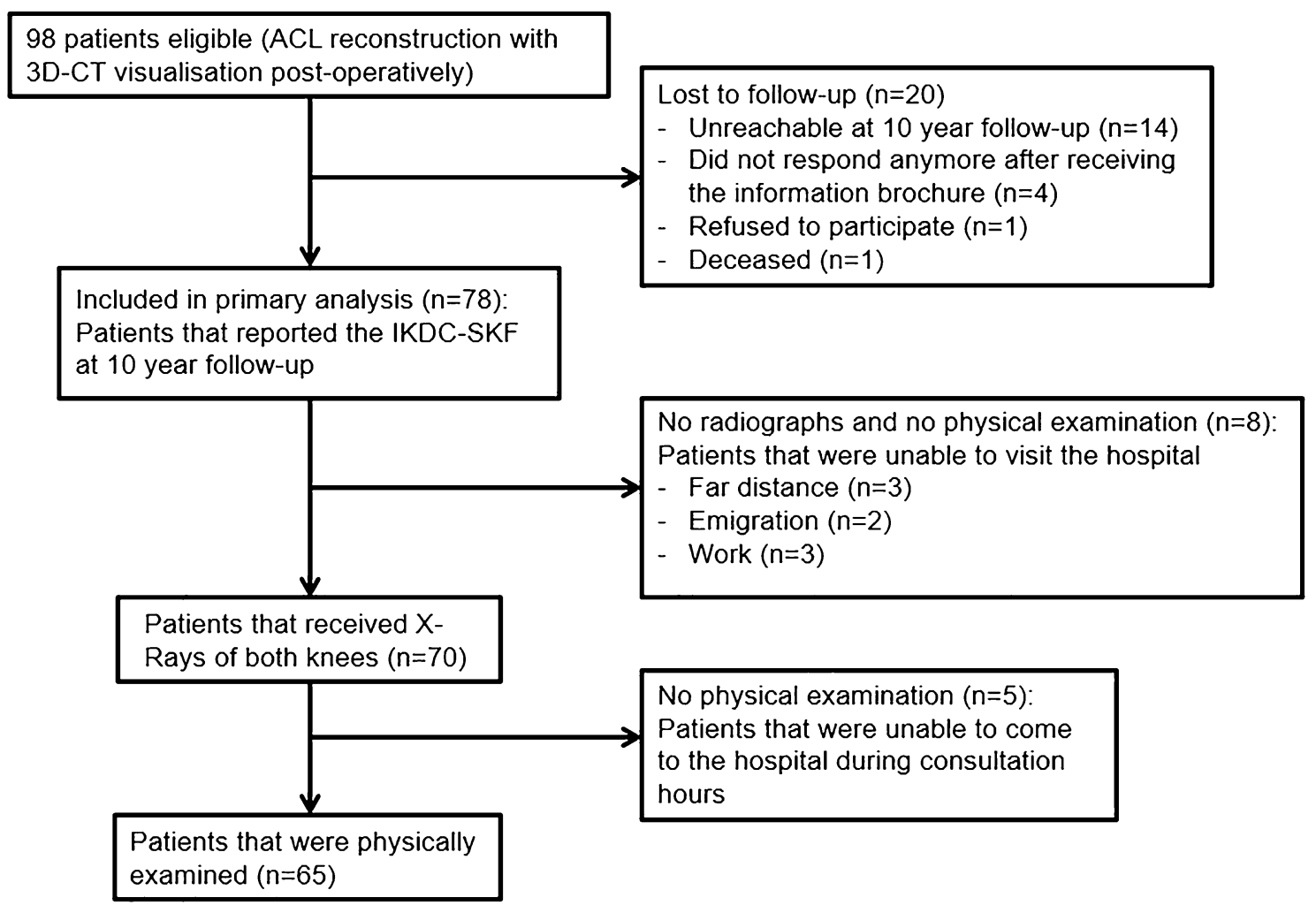

Fig. 2 Flow chart of the patients in the study

and exceeded the minimum clinically important difference of 11.5 points [18]. At 2-year follow-up, the IKDC-SKF score was highest, with a mean score of $85.8( \pm 13.3)$. At 10-year follow-up, 11 patients $(14.1 \%)$ had a graft failure and 9 patients had a contralateral ACL failure (11.5\%).

At 10 -year follow-up, 16 patients $(24.6 \%)$ were classified into the overall failure group (Table 3 ). In this group, 6 patients had BPTB grafts and 10 patients had hamstring grafts. The femoral posterior-to-anterior tunnel aperture position (tfem, $p=0.03$ ) showed a significant positive relation with overall failure. The mean posterior-to-anterior femoral tunnel aperture position (tfem) of the 'no failure' group was $37.7 \%$ and was $44.1 \%$ in the 'failure' group. Youden's index indicated that the cut-off point was at $35.0 \%$. Femoral tunnel apertures placed further anteriorly than $35 \%$ in posterior-to-anterior direction showed increased overall failure. Of the 16 overall failures, $15(93.8 \%)$ were placed further anteriorly than the cut-off point of $35 \%$. The femoral height positions and tibial tunnel aperture positions did not show significant relations with overall failure (Figs. 3B, 4B and Table 2).

\section{Secondary outcome}

We found no significant associations between the tunnel aperture positions and the development of osteoarthritis
(Table 2). Twenty-two patients (28.2\%) developed mild to severe osteoarthritis (K\&L grade 2 to 4 ) in the operated knee.

\section{Discussion}

This study shows that the femoral tunnel aperture position did significantly affect overall failure. The tibial tunnel aperture position did not significantly affect overall failure. The femoral and tibial tunnel aperture positions did not significantly affect the IKDC-SKF and the development of osteoarthritis at long-term follow-up. The overall patientreported and clinical outcome of ACL reconstruction was good to excellent.

The results of this study according to the IKDC-SKF are most comparable with the long-term follow-up study of Sundemo et al. In their follow-up of 16 years, no correlation between the femoral tunnel position and the IKDCSKF score was found either [50]. However, these longterm findings are in contrast with the short-term findings of other studies that demonstrate that anatomic tunnel positions show superior results over non-anatomic positions. These demonstrated that posteriorly placed femoral tunnels, ranging between 25 and $40 \%$ of the posterior-toanterior distance along Blumensaat, had positive effect on 
Table 1 Baseline and peroperative characteristics of patients $(n=98)$

\begin{tabular}{|c|c|c|c|}
\hline & Included $(n=78)$ & Lost to follow-up $(n=20)$ & $P$ value \\
\hline Age at operation, years & $24.9(21.0-30.1)$ & $27.0(21.6-31.5)$ & (n.s.) \\
\hline Gender, $n(\%)$ & & & (n.s.) \\
\hline Male & $58(74.4)$ & $17(85.0)$ & \\
\hline BMI at operation, $\mathrm{kg} / \mathrm{m}^{2}$ & $23.9(22.7-25.6)$ & $25.2(23.7-27.1)$ & (n.s.) \\
\hline Graft type, $n(\%)$ & & & (n.s.) \\
\hline ВРТВ & $33(42.3)$ & $13(65.0)$ & \\
\hline Hamstring & $45(57.7)$ & $7(35.0)$ & \\
\hline Type of surgery, $n(\%)$ & & & (n.s.) \\
\hline CAS & $36(46.2)$ & $9(45.0)$ & \\
\hline Conventional & $42(53.8)$ & $11(55.0)$ & \\
\hline \multicolumn{4}{|l|}{ Tunnel aperture positions } \\
\hline tfem & $39.1(9.4)$ & $40.4(9.6)$ & (n.s.) \\
\hline hfem & $38.5(9.0)$ & $38.7(9.9)$ & (n.s.) \\
\hline aptib & $38.8(6.3)$ & $37.8(6.4)$ & (n.s.) \\
\hline mltib & $42.4(4.8)$ & $44.0(4.7)$ & (n.s.) \\
\hline Meniscal tear per-operative, $n(\%)$ & & & (n.s.) \\
\hline No tear & $55(70.5)$ & $15(75.0)$ & \\
\hline Medial tear & $7(9.0)$ & $1(5.0)$ & \\
\hline Lateral tear & $15(19.2)$ & $4(20.0)$ & \\
\hline Combined & $1(1.3)$ & $0(0.0)$ & \\
\hline Chondral defect per-operative, $n(\%)$ & & & (n.s.) \\
\hline No defect & $53(67.9)$ & $14(70.0)$ & \\
\hline Patellar & $2(2.6)$ & $1(5.0)$ & \\
\hline Medial & $12(15.4)$ & $3(15.0)$ & \\
\hline Lateral & $4(5.1)$ & $1(5.0)$ & \\
\hline Combined & $7(9.0)$ & $1(5.0)$ & \\
\hline
\end{tabular}

tfem posterior-to-anterior femoral tunnel position, hfem superior-inferior femoral tunnel position, aptib anterior-to-posterior tibial tunnel position, mltib medial-to-lateral tibial tunnel position

Median value and (interquartile range) is presented for age and BMI

Mean value (standard deviation) is presented for the tunnel aperture positions

Frequency (percentage) is presented for the other characteristics patient-reported outcome [6, 21, 36, 43, 46]. Tibial tunnels ranging between 32 and $46 \%$ of the anterior-to-posterior distance of the plateau influenced patient-reported outcome positively $[4,36,43]$. A possible explanation for the absent relation between the reported outcome scores and the tunnel positions is the subjectivity of patient-reported outcome scores. Therefore, these scores are sensitive for interpretation bias. As could be observed in the questionnaires, some patients who reported low IKDC scores were paradoxically still able to participate in high-level or high-intensity sports. Vice versa, patients that have proven clinical graft laxity can still report excellent subjective outcome [12].

The femoral tunnel aperture positions did significantly affect the other primary outcome, overall failure. Femoral tunnel apertures placed further posteriorly in the femoral condyle showed a decrease in overall failure at longterm follow-up. This signifies the importance of posterior placement of the femoral tunnel apertures to resemble anatomy and to reduce failure rates. The cut-off point for this posterior zone was identified at $35.0 \%$. No significant association was found between femoral superior-anterior height and tunnel aperture position. This study therefore identifies the posterior $35 \%$ in posterior-to-anterior direction as a safe zone for femoral tunnel aperture placement. However, the surgeon should always take the risk of a posterior wall blowout into account when placing the femoral tunnel too close to the posterior articular margin [34]. Also, different studies do indicate the anatomical insertion of the ACL lies just above the midline in superior-inferior height $[28,60]$.

The decrease in overall failure is due to the anatomic resemblance of the original ACL of posteriorly placed femoral tunnels. Overall, anterior femoral tunnel positions lead to more vertically oriented grafts that allow more anteroposterior and rotational translation. This results in more 

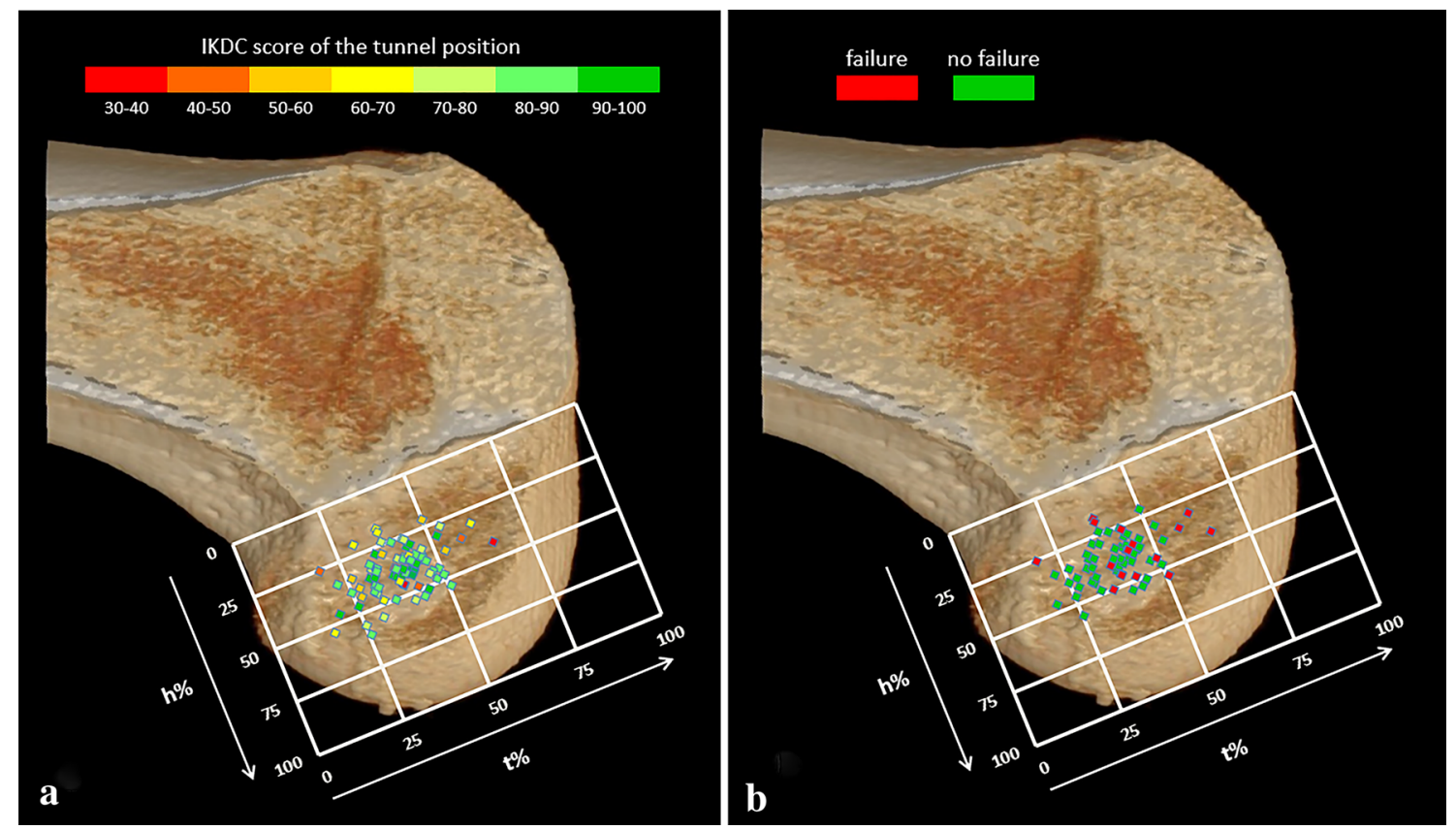

Fig. 3 Tunnel aperture positions in the femur. A displays the IKDC-SKF score of each aperture position. B displays per aperture position if overall failure was present or not present
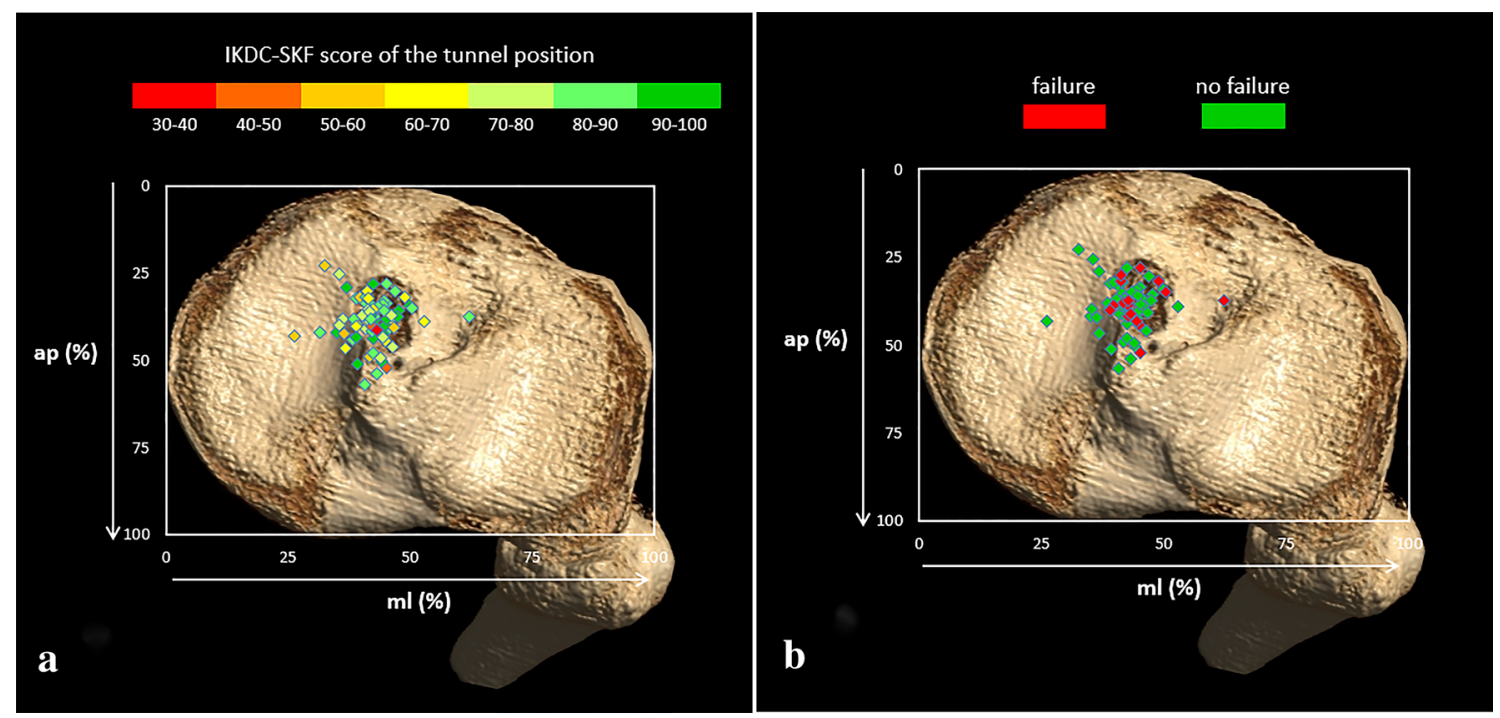

Fig. 4 Tunnel aperture positions in the tibia. A displays the IKDC-SKF score of each aperture position. B displays per aperture position if overall failure was present or not present

rotational instability and increases chondral and meniscal stress [1, 28, 41, 53, 59]. Overall, anterior femoral tunnel positions can also lead to graft impingement in the intercondylar roof $[28,54]$.

The findings on overall failure correspond with the shortterm findings of several studies. These studies reported that anatomical, more oblique graft positions gave superior clinical outcome, increased rotational stability and lower revision rates [3, 7, 21, 24, 43, 45, 53]. These findings also correspond with the long-term findings of Pinczewski et al., who state that more vertical inclination is associated with increased rotational instability [41]. However, the previously mentioned study of Sundemo et al. did not show a correlation with the femoral tunnel position and clinical outcome (e.g. Lachman, pivot shift test and KT-1000) [50]. This can be explained because they retrospectively assessed 
Table 2 Relations between tunnel aperture positions and primary outcomes

\begin{tabular}{lllll}
\hline & tfem & hfem & aptib & mltib \\
\hline $\begin{array}{l}\text { IKDC-SKF } n=78 \\
\text { B Coefficient }\end{array}$ & 0.05 & & & \\
$\begin{array}{l}95 \% \text { CI } \\
p \text { value }\end{array}$ & $(-0.38 ; 0.48)$ & $(-0.26 ; 0.67)$ & $(-0.56 ; 0.84)$ & $(-0.35 ; 1.32)$ \\
$\begin{array}{l}\text { Overall failure } n=65 \\
\text { Odds ratio }\end{array}$ & $($ n.s. $)$ & $($ n.s. $)$ & $($ n.s. $)$ & $($ n.s. $)$ \\
$\begin{array}{l}\text { 95\% CI } \\
p \text { value }\end{array}$ & 1.09 & 0.98 & & \\
$\begin{array}{l}\text { Osteoarthritis } n=70 \\
\text { Odds ratio }\end{array}$ & $(1.01 ; 1.18)$ & $(0.91 ; 1.06)$ & $(0.80 ; 1.04)$ & $(0.99 ; 1.39)$ \\
$\begin{array}{l}\text { 95\% CI } \\
p \text { value }\end{array}$ & 0.03 & $($ n.s. $)$ & $($ n.s. $)$ & $($ n.s. $)$ \\
\hline
\end{tabular}

tfem posterior-to-anterior femoral tunnel position, hfem superior-inferior femoral tunnel position, aptib anterior-to-posterior tibial tunnel position, mltib medial-to-lateral tibial tunnel position

The outcome variables presented are the IKDC-SKF, overall failure and osteoarthritis (italics)

$N$ describes the number of participants that were analysed per outcome variable

Adjusted values are presented
Table 3 Criteria for overall failure

\begin{tabular}{ll}
\hline Criterion & Number of cases \\
\hline Graft failure & $3(19 \%)$ \\
Knee laxity during physical examination & $5(31 \%)$ \\
IKDC-SKF $<50$ & $4(25 \%)$ \\
Multiple criteria ( $\geq 2$ criteria) & $4(25 \%)$ \\
Total overall failure & $16(100 \%)$ \\
\hline
\end{tabular}

Frequencies (percentages) are presented

the tunnel positions on radiographs. Therefore, they had to exclude graft ruptures and contralateral ruptures from their study. In this study, the tunnel positions were determined directly post-operative and ruptures were included in the overall failure group.

The tibial tunnel aperture positions did not affect overall failure, which is contradictive to the findings of Inderhaug et al. [15]. Their study suggests that posteriorly placed tunnels ( $>50 \%$ of anterior-to-posterior distance) show increased rotational instability. The absence of a tibial relation with overall failure could be explained by the relatively small range tibial anterior-to-posterior tunnel aperture positions. The tibial aperture positions in our study ranged until $56.9 \%$ of the anterior-to-posterior distance with a mean of $38.8 \%$, whilst Inderhaug et al. only called placement too posteriorly if it was over $50 \%$. Inderhaug et al. also assessed the tunnel positions on radiographs, which offers a less precise visualisation compared to 3D-CT $[25,30]$. However, this study did find a non-significant trend on overall failure according medialto-lateral tunnel positions. This trend indicates that more laterally placed tunnels show increased overall failure. This trend can again be explained by the instability and inferior results associated with more vertically oriented grafts [24, 43, 53].

Osteoarthritis did not show a significant relation with tunnel aperture positions. This corresponds with the findings of the long-term study from Sundemo et al. [50]. The prevalence of osteoarthritis $(31.0 \%)$ was lower compared to other long-term follow-up [37, 49]. The prevalence of graft failures was higher than the prevalence described in the literature [27]. This can be caused by our definition of graft failures, which also includes partially ruptured grafts and need for revision surgery.

In general, ACL reconstruction provided favourable increases of both short- and long-term patient-reported outcome that were significant and clinically relevant, similar to other cohorts [13, 44]. The decrease in IKDC-SKF between 2- and 10-year follow-up can be caused by multiple factors, such as lower activity levels because of the negative effect of ageing and the occurrence of comorbidities [29].

3D-CT was used to assess the tunnel positions directly post-operative. $3 \mathrm{D}-\mathrm{CT}$ is the most accurate measurement tool with the highest inter-observer ICC $(>0.993)$ and intraobserver ICC $(>0.963)$. Therefore, the established anatomic coordinate axis method 3D-CT provides the most reliable representation of the anatomy $[25,30,52]$. This is particularly important in visualising the femoral condyle with its convex shape. This was the first study that coupled directly visualised tunnel positions by 3D-CT to long-term outcome. Besides that, this study was amongst the first studies that coupled tunnel positioning in general to long-term outcome. 
There were limitations present in this study. All patients in this study were operated with the trans-tibial technique. Several studies have shown that the anteromedial technique achieves a more anatomic tunnel placement, improved antero-posterior and rotational knee stability [10, 26, 33, $38,42,48,51,58]$. However, there is inconsistency between these different studies if the improved graft anatomy of the anteromedial technique leads to better reported and clinical outcome. Although there was adjusted for possible confounders, there was not adjusted for patient-specific morphologic knee differences. Because generalised figures of 3D-CT were used, some tunnel apertures appear to be positioned outside of the anatomic boundaries (e.g. in the medial tibial plateau) (Figs. 3 and 4). There were indeed outliers in tunnel positions, but these were still positioned within the anatomic boundaries of the individuals' tibia and femur. The outliers were mostly due to anatomical variations [16, $19,35]$. This study analysed both the femoral tunnel position and tibial tunnel position as two independent parameters but it is clear that these positions influence each other. The choice of independent assessment was based on multiple studies that also assessed the tunnel positions independently $[3,4,9,15,16,20,21,36,50]$. The number of patients that were physically examined was smaller because some patients were unable to come to the hospital. Therefore, the overall failure and osteoarthritis group had a reduced sample size, the IKDC-SKF score did not have a reduced sample size. Since there was a fixed number of study participants because it was a follow-up, there was no power analysis performed to determine the sample size. This was an exploratory study that researched the long-term effects of a relative spread of tunnel positions. The results of this study offer guidance to surgeons to operate more precisely and accurately and reconstruct a long-lasting graft. Future research that compares the effect that anatomical versus nonanatomical tunnel positions have on long-term outcome is required to confirm the findings of this study.

\section{Conclusion}

Femoral and tibial tunnel positions were not associated with long-term patient-reported outcome and radiographic osteoarthritis. Long-term overall failure was more frequently seen in patients with a more anteriorly placed femoral tunnel. This study identified a safe zone located at the most posterior $35 \%$ of the femoral condyle parallel to Blumensaat.

Funding No funding was received for this paper.

\section{Declarations}

Conflict of interest The authors declares that they have no conflict of interest.

Ethical Approval The Medical Ethics Committee of the Erasmus University Medical Centre approved the study protocol of the RCT and the follow-up study (METC-2006-223 and METC-2019-0369).

Open Access This article is licensed under a Creative Commons Attribution 4.0 International License, which permits use, sharing, adaptation, distribution and reproduction in any medium or format, as long as you give appropriate credit to the original author(s) and the source, provide a link to the Creative Commons licence, and indicate if changes were made. The images or other third party material in this article are included in the article's Creative Commons licence, unless indicated otherwise in a credit line to the material. If material is not included in the article's Creative Commons licence and your intended use is not permitted by statutory regulation or exceeds the permitted use, you will need to obtain permission directly from the copyright holder. To view a copy of this licence, visit http://creativecommons.org/licenses/by/4.0/.

\section{References}

1. Abebe ES, Utturkar GM, Taylor DC, Spritzer CE, Kim JP, Moorman CT 3rd et al (2011) The effects of femoral graft placement on in vivo knee kinematics after anterior cruciate ligament reconstruction. J Biomech 44:924-929

2. Achtnich A, Ranuccio F, Willinger L, Pogorzelski J, Imhoff AB, Braun S et al (2018) High incidence of partially anatomic tunnel placement in primary single-bundle ACL reconstruction. Knee Surg Sports Traumatol Arthrosc 26:462-467

3. Ahn JH, Kim JD, Kang HW (2015) Anatomic placement of the femoral tunnels in double-bundle anterior cruciate ligament reconstruction correlates with improved graft maturation and clinical outcomes. Arthroscopy 31:2152-2161

4. Avadhani A, Rao PS, Rao SK (2010) Effect of tibial tunnel position on arthroscopically assisted anterior cruciate ligament reconstruction using bone-patellar tendon-bone grafts: a prospective study. Singapore Med J 51:413-417

5. Bernard M, Hertel P, Hornung H, Cierpinski T (1997) Femoral insertion of the ACL. Radiographic quadrant method. Am J Knee Surg 10:14-21 (discussion 21-12)

6. Biswal UK, Balaji G, Nema S, Poduval M, Menon J, Patro DK (2016) Correlation of tunnel widening and tunnel positioning with short-term functional outcomes in single-bundle anterior cruciate ligament reconstruction using patellar tendon versus hamstring graft: a prospective study. Eur J Orthop Surg Traumatol 26:647-655

7. Byrne KJ, Hughes JD, Gibbs C, Vaswani R, Meredith SJ, Popchak A et al (2021) Non-anatomic tunnel position increases the risk of revision anterior cruciate ligament reconstruction. Knee Surg Sports Traumatol Arthrosc. https://doi.org/10.1007/ s00167-021-06607-7

8. de Padua VBC, Saithna A, Chagas EFB, Zutin TLM, Piazzalunga LF, Patriarcha LF et al (2021) Rate of tibial tunnel malposition is not changed by drilling entirely within the stump of preserved remnants during ACL reconstruction: a prospective comparative 3D-CT study. Orthop J Sports Med 9:23259671211037324

9. Fernandes TL, Fregni F, Weaver K, Pedrinelli A, Camanho GL, Hernandez AJ (2014) The influence of femoral tunnel position in 
single-bundle ACL reconstruction on functional outcomes and return to sports. Knee Surg Sports Traumatol Arthrosc 22:97-103

10. Fernandes TL, Moreira HH, Andrade R, Sasaki SU, Bernardo WM, Pedrinelli A et al (2021) Clinical outcome evaluation of anatomic anterior cruciate ligament reconstruction with tunnel positioning using gold standard techniques: a systematic review and meta-analysis. Orthop J Sports Med 9:23259671211013330

11. Forsythe B, Kopf S, Wong AK, Martins CA, Anderst W, Tashman $S$ et al (2010) The location of femoral and tibial tunnels in anatomic double-bundle anterior cruciate ligament reconstruction analyzed by three-dimensional computed tomography models. J Bone Joint Surg Am 92:1418-1426

12. Goodwillie AD, Shah SS, McHugh MP, Nicholas SJ (2017) The effect of postoperative KT-1000 arthrometer score on long-term outcome after anterior cruciate ligament reconstruction. Am J Sports Med 45:1522-1528

13. Group MK, Spindler KP, Huston LJ, Chagin KM, Kattan MW, Reinke EK et al (2018) Ten-year outcomes and risk factors after anterior cruciate ligament reconstruction: a MOON longitudinal prospective cohort study. Am J Sports Med 46:815-825

14. Higgins LD, Taylor MK, Park D, Ghodadra N, Marchant M, Pietrobon R et al (2007) Reliability and validity of the International Knee Documentation Committee (IKDC) subjective knee form. Joint Bone Spine 74:594-599

15. Inderhaug E, Strand T, Fischer-Bredenbeck C, Solheim E (2014) Effect of a too posterior placement of the tibial tunnel on the outcome 10-12 years after anterior cruciate ligament reconstruction using the 70-degree tibial guide. Knee Surg Sports Traumatol Arthrosc 22:1182-1189

16. Iriuchishima T, Goto B, Ryu K, Fu FH (2019) The Blumensaat's line morphology influences to the femoral tunnel position in anatomical ACL reconstruction. Knee Surg Sports Traumatol Arthrosc 27:3638-3643

17. Iriuchishima $\mathrm{T}$, Horaguchi $\mathrm{T}$, Kubomura $\mathrm{T}$, Morimoto $\mathrm{Y}, \mathrm{Fu} \mathrm{FH}$ (2011) Evaluation of the intercondylar roof impingement after anatomical double-bundle anterior cruciate ligament reconstruction using 3D-CT. Knee Surg Sports Traumatol Arthrosc 19:674-679

18. Irrgang JJ, Anderson AF, Boland AL, Harner CD, Neyret P, Richmond JC et al (2006) Responsiveness of the International Knee Documentation Committee subjective knee form. Am J Sports Med 34:1567-1573

19. Iwasaki K, Inoue M, Kasahara Y, Tsukuda K, Kawahara H, Yokota I et al (2020) Inclination of Blumensaat's line influences on the accuracy of the quadrant method in evaluation for anterior cruciate ligament reconstruction. Knee Surg Sports Traumatol Arthrosc 28:1885-1893

20. Jepsen CF, Lundberg-Jensen AK, Faunoe P (2007) Does the position of the femoral tunnel affect the laxity or clinical outcome of the anterior cruciate ligament-reconstructed knee? A clinical, prospective, randomized, double-blind study. Arthroscopy 23:1326-1333

21. Khalfayan EE, Sharkey PF, Alexander AH, Bruckner JD, Bynum EB (1996) The relationship between tunnel placement and clinical results after anterior cruciate ligament reconstruction. Am J Sports Med 24:335-341

22 Kohn MD, Sassoon AA, Fernando ND (2016) Classifications in brief: Kellgren-Lawrence classification of osteoarthritis. Clin Orthop Related Res® 474:1886-1893

23. Kosy JD, Walmsley K, Gordon EA, Heddon SV, Anaspure R, Schranz PJ et al (2021) Remnant preservation does not affect accuracy of tibial tunnel positioning in single-bundle ACL reconstruction. Knee Surg Sports Traumatol Arthrosc 29:1157-1163

24. Lee MC, Seong SC, Lee S, Chang CB, Park YK, Jo H et al (2007) Vertical femoral tunnel placement results in rotational knee laxity after anterior cruciate ligament reconstruction. Arthroscopy 23:771-778

25. Lertwanich $P$, Martins CA, Asai S, Ingham SJ, Smolinski P, Fu FH (2011) Anterior cruciate ligament tunnel position measurement reliability on 3-dimensional reconstructed computed tomography. Arthroscopy 27:391-398

26. Loucas M, Loucas R, D’Ambrosi R, Hantes ME (2021) Clinical and radiological outcomes of anteromedial portal versus transtibial technique in ACL reconstruction: a systematic review. Orthop J Sports Med 9:23259671211024590

27. Magnussen RA, Meschbach NT, Kaeding CC, Wright RW, Spindler KP (2015) ACL graft and contralateral ACL tear risk within ten years following reconstruction: a systematic review. JBJS Reviews 3:e3. https://doi.org/10.2106/JBJS.RVW.N.00052

28. Markatos K, Kaseta MK, Lallos SN, Korres DS, Efstathopoulos $\mathrm{N}$ (2013) The anatomy of the ACL and its importance in ACL reconstruction. Eur J Orthop Surg Traumatol 23:747-752

29. Marx RG, Stump TJ, Jones EC, Wickiewicz TL, Warren RF (2001) Development and evaluation of an activity rating scale for disorders of the knee. Am J Sports Med 29:213-218

30. Meuffels DE, Potters JW, Koning AH, Brown CH Jr, Verhaar JA, Reijman M (2011) Visualization of postoperative anterior cruciate ligament reconstruction bone tunnels: reliability of standard radiographs, CT scans, and 3D virtual reality images. Acta Orthop 82:699-703

31. Meuffels DE, Reijman M, Verhaar JA (2012) Computer-assisted surgery is not more accurate or precise than conventional arthroscopic ACL reconstruction: a prospective randomized clinical trial. J Bone Joint Surg Am 94:1538-1545

32. Miller MD, Olszewski AD (1997) Posterior tibial tunnel placement to avoid anterior cruciate ligament graft impingement by the intercondylar roof. An in vitro and in vivo study. Am J Sports Med 25:818-822

33. Minguell J, Nuñez JH, Reverte-Vinaixa MM, Sallent A, GargalloMargarit A, Castellet E (2019) Femoral tunnel position in chronic anterior cruciate ligament rupture reconstruction: randomized controlled trial comparing anatomic, biomechanical and clinical outcomes. Eur J Orthop Surg Traumatol 29:1501-1509

34. Mitchell JJ, Dean CS, Chahla J, Menge TJ, Cram TR, LaPrade RF (2016) Posterior wall blowout in anterior cruciate ligament reconstruction: a review of anatomic and surgical considerations. Orthop J Sports Med 4:2325967116652122

35. Miyaji N, Araki D, Hoshino Y, Kanzaki N, Nagai K, Matsumoto $T$ et al (2021) The sagittal cutting plane affects evaluation of the femoral bone tunnel position on three-dimensional computed tomography after anterior cruciate ligament reconstruction. Knee Surg Sports Traumatol Arthrosc 29:398-404

36. Moisala AS, Jarvela T, Harilainen A, Sandelin J, Kannus P, Jarvinen M (2007) The effect of graft placement on the clinical outcome of the anterior cruciate ligament reconstruction: a prospective study. Knee Surg Sports Traumatol Arthrosc 15:879-887

37. Oiestad BE, Holm I, Aune AK, Gunderson R, Myklebust G, Engebretsen L et al (2010) Knee function and prevalence of knee osteoarthritis after anterior cruciate ligament reconstruction: a prospective study with 10 to 15 years of follow-up. Am J Sports Med 38:2201-2210

38. Osti M, Krawinkel A, Ostermann M, Hoffelner T, Benedetto KP (2015) Femoral and tibial graft tunnel parameters after transtibial, anteromedial portal, and outside-in single-bundle anterior cruciate ligament reconstruction. Am J Sports Med 43:2250-2258

39. Parkar AP, Adriaensen M, Vindfeld S, Solheim E (2017) The Anatomic centers of the femoral and tibial insertions of the anterior cruciate ligament: a systematic review of imaging and cadaveric studies reporting normal center locations. Am J Sports Med 45:2180-2188 
40. Pedneault C, Laverdière C, Hart A, Boily M, Burman M, Martineau PA (2019) Evaluating the accuracy of tibial tunnel placement after anatomic single-bundle anterior cruciate ligament reconstruction. Am J Sports Med 47:3187-3194

41. Pinczewski LA, Salmon LJ, Jackson WF, von Bormann RB, Haslam PG, Tashiro S (2008) Radiological landmarks for placement of the tunnels in single-bundle reconstruction of the anterior cruciate ligament. J Bone Joint Surg Br 90:172-179

42. Rahardja R, Zhu M, Love H, Clatworthy MG, Monk AP, Young SW (2020) No difference in revision rates between anteromedial portal and transtibial drilling of the femoral graft tunnel in primary anterior cruciate ligament reconstruction: early results from the New Zealand ACL Registry. Knee Surg Sports Traumatol Arthrosc 28:3631-3638

43. Sadoghi P, Kropfl A, Jansson V, Muller PE, Pietschmann MF, Fischmeister MF (2011) Impact of tibial and femoral tunnel position on clinical results after anterior cruciate ligament reconstruction. Arthroscopy 27:355-364

44. Salmon LJ, Russell VJ, Refshauge K, Kader D, Connolly C, Linklater J et al (2006) Long-term outcome of endoscopic anterior cruciate ligament reconstruction with patellar tendon autograft: minimum 13-year review. Am J Sports Med 34:721-732

45. Seo SS, Kim CW, Lee CR, Kwon YU, Kim MW, Kim OG et al (2019) Effect of femoral tunnel position on stability and clinical outcomes after single-bundle anterior cruciate ligament reconstruction using the outside-in technique. Arthroscopy 35:1648-1655

46. Sommer C, Friederich NF, Muller W (2000) Improperly placed anterior cruciate ligament grafts: correlation between radiological parameters and clinical results. Knee Surg Sports Traumatol Arthrosc 8:207-213

47. Staubli HU, Rauschning W (1994) Tibial attachment area of the anterior cruciate ligament in the extended knee position. Anatomy and cryosections in vitro complemented by magnetic resonance arthrography in vivo. Knee Surg Sports Traumatol Arthrosc 2:138-146

48. Stone AV, Chahla J, Manderle BJ, Beletsky A, Bush-Joseph CA, Verma NN (2020) ACL reconstruction graft angle and outcomes: transtibial vs anteromedial reconstruction. HSS J 16:256-263

49. Struewer J, Frangen TM, Ishaque B, Bliemel C, Efe T, Ruchholtz $\mathrm{S}$ et al (2012) Knee function and prevalence of osteoarthritis after isolated anterior cruciate ligament reconstruction using bone-patellar tendon-bone graft: long-term follow-up. Int Orthop 36:171-177

50. Sundemo D, Martensson J, HamrinSenorski E, Svantesson E, Kartus J, Sernert N et al (2019) No correlation between femoral tunnel orientation and clinical outcome at long-term follow-up after nonanatomic anterior cruciate ligament reconstruction. Knee Surg Sports Traumatol Arthrosc 27:3400-3410

51. Tampere T, Devriendt W, Cromheecke M, Luyckx T, Verstraete M, Victor J (2019) Tunnel placement in ACL reconstruction surgery: smaller inter-tunnel angles and higher peak forces at the femoral tunnel using anteromedial portal femoral drilling-a 3D and finite element analysis. Knee Surg Sports Traumatol Arthrosc 27:2568-2576

52. Tank S, Dutt S, Sehrawat R, Kumar V, Sabat D (2021) 3D CT evaluation of femoral and tibial tunnels in anatomic double bundle anterior cruciate ligament reconstruction. J Clin Orthop Trauma 15:22-26

53. Tashiro Y, Okazaki K, Murakami K, Matsubara H, Osaki K, Iwamoto $Y$ et al (2017) Anterolateral rotatory instability in vivo correlates tunnel position after anterior cruciate ligament reconstruction using bone-patellar tendon-bone graft. World J Orthop 8:913-921

54. Thein R, Spitzer E, Doyle J, Khamaisy S, Nawabi DH, Chawla H et al (2016) The ACL graft has different cross-sectional dimensions compared with the native ACL: implications for graft impingement. Am J Sports Med 44:2097-2105

55. Udagawa K, Niki Y, Enomoto H, Toyama Y, Suda Y (2014) Factors influencing graft impingement on the wall of the intercondylar notch after anatomic double-bundle anterior cruciate ligament reconstruction. Am J Sports Med 42:2219-2225

56. van Meer BL, Meuffels DE, Vissers MM, Bierma-Zeinstra SM, Verhaar JA, Terwee CB et al (2013) Knee injury and Osteoarthritis Outcome Score or International Knee Documentation Committee Subjective Knee Form: which questionnaire is most useful to monitor patients with an anterior cruciate ligament rupture in the short term? Arthroscopy 29:701-715

57. Vignos MF, Smith CR, Roth JD, Kaiser JM, Baer GS, Kijowski R et al (2020) Anterior cruciate ligament graft tunnel placement and graft angle are primary determinants of internal knee mechanics after reconstructive surgery. Am J Sports Med 48:3503-3514

58. Vijayan S, Kyalakond H, Kulkarni MS, Aroor MN, Shetty S, Bhat V et al (2021) Clinical outcome of anterior cruciate ligament reconstruction with modified transtibial and anteromedial portal. Musculoskelet Surg. https://doi.org/10.1007/s12306-021-00727-6

59. Westermann RW, Wolf BR, Elkins J (2017) Optimizing graft placement in anterior cruciate ligament reconstruction: a finite element analysis. J Knee Surg 30:97-106

60. Xu H, Zhang C, Zhang Q, Du T, Ding M, Wang Y et al (2016) A systematic review of anterior cruciate ligament femoral footprint location evaluated by quadrant method for single-bundle and double-bundle anatomic reconstruction. Arthroscopy 32:1724-1734

Publisher's Note Springer Nature remains neutral with regard to jurisdictional claims in published maps and institutional affiliations. 